\title{
UMA FORMULAÇÃo EXPLÍCITA PARA A SOLUÇÃo dE ESCOAMENTOS EM ÁGUAS RASAS
}

\author{
Denise Maria Varella Martinez e Maria Angela Vaz dos Santos \\ Fundação Universidade do Rio Grande - Curso de Pós-Graduação em Engenharia Ocêanica \\ Caixa Postal 474 - CEP 96201-900 Rio Grande, RS \\ smtdmvm@super.furg.bre angela@dmc.furg.br
}

\section{RESUMO}

No presente trabalho apresenta-se um algoritmo numérico para simular o escoamento bidimensional de um fluido incompressível, utilizando a formulação geral das equações de águas rasas, escritas na sua forma conservativa, capaz de auxiliar na solução de diversos problemas de engenharia costeira e ambiental. A técnica de elementos finitos é usada na discretização espacial do domínio do fluido.

O algoritmo está baseado em um procedimento explícito de integração no tempo, utilizando o esquema de Taylor-Galerkin, que explora as propriedades conservativas das equações governantes e incorpora um tratamento preciso dos termos convectivos. É empregada opcionalmente uma descrição mista de Euler-Lagrange na solução deste algoritmo.

Exemplos são analisados para mostrar a validação do modelo, para que a sua aplicação futura seja confiável.

\section{INTRODUÇÃO}

A solução das equações de águas rasas é de considerável importância para uma variedade de problemas de engenharia costeira e ambiental. O estudo nessa área é motivado pela necessidade de prever certos fenômenos ambientais e as alterações que podem ocorrer na natureza quando são construídos portos, barragens, comportas, molhes ou quando são emitidos efluentes térmicos e químicos.

$\mathrm{Na}$ análise dos problemas de engenharia oceânica a aplicação das equações de águas rasas, para modelar escoamentos em zonas de estuários, costeiras e lagos, tem atraído um crescente interesse da comunidade científica. O impacto ambiental e o aumento de obras de engenhara nessas zonas tem tornado de grande importância a simulação do escoamento. Uma das principais finalidades desta simulação é garantir uma locali- zação segura para tais obras, preservando o meio ambiente.

Usualmente, são empregadas formas linearizadas das equações de águas rasas, que não descrevem a evolução de processos físicos relevantes que ocorrem nos corpos d'água. Para a solução de uma formulação mais geral, que inclui efeitos não lienares, torna-se necessário recorrer a métodos numéricos tais como o método de diferenças finitas (MDF) e o método de elementos finitos (MEF).

A utilização do MEF na simulação de escoamentos em águas rasas na sua forma transiente e não linear (introduzindo os termos convectivos e de fricção no fundo) datam do início da década de 70 .

O esquema de Taylor-Galerkin usando o MEF tem dado uma notável contribuição no campo de problemas de escoamento compressíveis, e tem sido estendido para a solução de problemas em águas rasas. O método de Taylor-Galerkin é uma extensão da técnica de diferenças finitas de alta ordem desenvolvida originalmente por LaxWendroff e foi introduzido no contexto das equações de águas rasas por Kawahara (1978).

Entre os trabalhos que se destacam na aplicação do esquema de Taylor-Galerkin às equações de águas rasas, sobressai o realizado por Peraire et al. (1986), que utilizaram um modelo de elementos finitos para as equações de águas rasas na forma conservativa, utilizando o elemento triangular linear e o algoritmo de dois passos de tempo citado acima. Esta metodologia é capaz de resolver uma enorme classe de problemas de engenharia com custo computacional razoável.

O modelo desenvolvido, neste trabalho, objetiva simular escoamentos bidimensionais de fluidos incompressíveis, usando uma formulação geral das equações de águas rasas (Peraire et al., 1986), e tendo como base o algoritmo numérico para escoamento de fluidos compressíveis proposto por Vaz dos Santos (1993), que explora as similaridades entre as equações que governam ambos escoamentos.

O meio fluido é discretizado usando o método de elementos finitos com elementos lineares de três nós no plano. 
As equações que governam o escoamento são as equações de águas rasas obtidas pela integração na vertical das equações de Navier-Stokes para um fluido incompressível e num processo isotérmico. As equações são estabelecidas considerando uma descrição arbitrária lagrangeana-euleriana (ALE) (Donea et al., 1982). Esta descrição permite que a malha de elementos finitos acompanhe os contornos móveis ou o movimento da estrutura nos casos de interação fluido-estrutura. A integração das mesmas é realizada pelo método explícito de TaylorGalerkin (Donéa, 1984; Lõhner et al., 1984). O procedimento é adequado à implementação na moderna geração de computadores com processadores vetoriais e facilidades de paralelização.

Um modelo de viscosidade artificial (Löhner et al., 1985) é utilizado com o objetivo de estabilizar a solução numérica, no caso de problemas envolvendo escoamentos supercríticos, onde ocorrem oscilações nas proximidades dos saltos hidráulicos.

Exemplos são solucionados e comparados com resultados apresentados na literatura, a fim de validar o modelo.

\section{AS EQUAÇÕES DE ÁGUAS RASAS EM UMA DESCRIÇÃO ALE}

As equações de águas rasas em uma descrição mista de Euler-Lagrange, escritas na forma matricial compacta, apresentam-se como:

$$
\frac{\partial \mathrm{U}}{\partial \mathrm{t}}+\frac{\partial \mathrm{F}_{\mathrm{i}}}{\partial \mathbf{x}_{\mathrm{i}}}=\mathbf{R}_{\mathrm{S}}+\frac{\partial \mathbf{R}_{\mathrm{di}}}{\partial \mathbf{x}_{\mathrm{i}}}+\mathrm{w}_{\mathrm{i}} \frac{\partial \mathrm{U}}{\partial \mathbf{x}_{\mathrm{i}}}(\mathbf{i}=1,2)
$$

sendo,

$$
\begin{gathered}
U=\left[\begin{array}{c}
h \\
h u_{1} \\
h u_{2}
\end{array}\right], F_{1}=\left[\begin{array}{c}
h u_{1} \\
h u_{1} u_{1}+\frac{1}{2} g\left(h^{2}-H^{2}\right) \\
h u_{2} u_{1}
\end{array}\right] \\
F_{2}=\left[\begin{array}{c}
h u_{2} \\
h u_{1} u_{2} \\
h u_{2} u_{2}+\frac{1}{2} g\left(h^{2}-H^{2}\right)
\end{array}\right], \\
R_{8}=\left[\begin{array}{c}
0 \\
f h u_{2}+g(h-H) \frac{\partial H}{\partial x_{1}}+\frac{\tau_{1}}{\rho}-\frac{g u u_{1}}{c^{2}}-\frac{h}{\rho} \frac{\partial p_{s}}{\partial x_{1}} \\
-f h u_{1}+g(h-H) \frac{\partial H}{\partial x_{2}}+\frac{\tau_{2}}{\rho}-\frac{g u_{2} u_{2}}{c^{2}}-\frac{h}{\rho} \frac{\partial p_{2}}{\partial x_{2}}
\end{array}\right],
\end{gathered}
$$

$$
R_{d_{1}}=\left[\begin{array}{c}
0 \\
\frac{2 \mu_{1} h}{\rho} \frac{\partial u_{1}}{\partial x_{1}} \\
\frac{\mu_{1} h}{\rho}\left(\frac{\partial u_{2}}{\partial x_{1}}+\frac{\partial u_{1}}{\partial x_{2}}\right)
\end{array}\right], R_{d_{2}}=\left[\begin{array}{c}
0 \\
\frac{\mu_{-} h}{\rho}\left(\frac{\partial u_{2}}{\partial x_{1}}+\frac{\partial u_{1}}{\partial x_{2}}\right) \\
\frac{2 \mu_{4} h}{\rho} \frac{\partial u_{2}}{\partial x_{2}}
\end{array}\right]
$$

onde $\mathrm{h}$ é a profundidade total do fluido, $\mathrm{H}$ é a profundidade medida com relação ao nível médio, $u_{i}$ e $w_{i}$ são as componentes da velocidade promediada na vertical e da velocidade da malha no sistema de coordenadas $x_{i}, g$ é a aceleração da gravidade e $\rho$ a massa específica, $C$ e $\mu_{H}$ são o coeficiente de Chezy e o coeficiente de viscosidade turbulenta, respectivamente, $\tau_{1}$ e $\tau_{2}$ são as tensões de cisalhamento na superfície devida ao vento, $\mathrm{p}_{\mathrm{a}}$ é a pressão atmosférica, e f o coeficiente de Coriolis.

\section{ALGORITMO PARA A ANÁlISE DO FLUIDO}

\section{O método de Taylos-Galerkin de dois passos explícito}

As equações de águas rasas escritas em uma descrição ALE são integradas utilizando o método explícito de Taylor-Galerkin. O método de TaylorGalerkin é uma extensão da técnica de diferenças finitas de alta ordem desenvolvida originalmente por Lax-Wendroff, como foi citado anteriormente. O mesmo consiste em discretizar o problema no domínio do tempo através de uma expansão em série de Taylor das equações de conservação, e após realizar uma discretização espacial usando a técnica de Galerkin. A aplicação do método é conduzida em dois passos sob a Equação (1), como descritos a seguir.

Primeiro passo - Os valores das variáveis de campo no vetor U são levadas do instante de tempo $t_{n}$ ao instante de tempo $t_{n+1 / 2}$, utilizando uma expansão em série de Taylor, de primeira ordem, do vetor $U$ em $t_{n}=t$ com relação ao tempo:

$$
U^{n+1 / 2}=U^{n}+\left.\frac{1}{2} \Delta t \frac{\partial U^{n}}{\partial t}\right|^{n}+\theta\left(\Delta t^{2}\right)
$$

onde, o índice superior $\mathrm{n}$ indica uma avaliação no tempo $\mathrm{t}=\mathrm{t}_{\mathrm{n}}$. Considerando que:

$$
\frac{\partial \mathrm{U}}{\partial t}=\mathrm{R}_{\mathrm{S}}+\frac{\partial \mathrm{R}_{\mathrm{di}}}{\partial \mathrm{x}_{\mathrm{i}}}-\frac{\partial \mathrm{F}_{\mathrm{i}}}{\partial \mathrm{x}_{\mathrm{i}}}+\mathrm{w}_{\mathrm{i}} \frac{\partial \mathrm{U}}{\partial \mathrm{x}_{\mathrm{i}}}
$$


então,

$U^{n+1 / 2}=U^{n}-\frac{1}{2} \Delta t\left[-R_{8 j}+\frac{\partial F_{i}}{\partial x_{i}}-\frac{\partial R_{d_{i}}}{\partial x_{i}}-w_{i} \frac{\partial U}{\partial x_{i}}\right]$

Indicando a função de forma linear no elemento associado com o nó j por $\mathrm{N}_{\mathrm{i}}$ e a função de forma constante associada com o elemento $\mathrm{E}$ por $P_{E}$, as aproximações para as variáveis envolvidas no problema escritas na forma padrão de elementos finitos (Burnett, 1987) são:

$$
\begin{array}{lc}
U^{n}=\sum_{j} N_{U_{i}} ; & w_{i}^{n}=\sum_{j} N_{j} w_{i j}^{n} \\
F_{i}^{n}=\sum_{j} N_{j} F_{i j}^{n} ; & u^{n+1 / 2}=\sum_{E} P_{E} U_{E}^{n+1 / 2} \\
R_{d}^{n}=\sum_{j} N_{j} R_{d j}^{n} ; & R_{g}^{n} \approx \sum_{j} N_{j} R_{g j}^{n} \\
& (i=1,2 j=1,2,3)
\end{array}
$$

Aplicando à Equação (4) a forma residual ponderada de Galerkin (Chung, 1978), obtém-se:

$$
\begin{aligned}
& \sum_{E}\left(\int_{\Omega} P_{E} P_{E} d \Omega\right) U_{E}^{n+1 / 2}=\left[\sum_{j}\left(\int_{\Omega} N_{j} P_{E} d \Omega\right) U_{j}^{n}\right] \\
& -\frac{1}{2} \Delta t\left[\sum\left(\int_{\alpha} \frac{\partial \mathbb{N}_{j}}{\partial x_{i}} P_{E} d \Omega\right)\left[\left(E i^{n}-R_{d j}^{n}\right)+\text { termos relativos } a\left(W_{i} \frac{\partial U}{\partial x_{i}}\right)\right]\right] \\
& +\frac{1}{2} \Delta t\left[\sum_{j}\left(h_{\Omega} N_{j} d \Omega\right) R_{S i}^{n}\right]
\end{aligned}
$$

onde, segundo proposta apresentada em Vaz dos Santos (1993), os termos relativos a $w_{i} \frac{\partial U}{\partial x_{i}}$ podem; ser representados como $\sum_{j}\left(\int_{\Omega} \frac{\partial \mathrm{N}_{\mathrm{j}}}{\partial \mathrm{x}_{\mathrm{i}}} \mathrm{P}_{\mathrm{E}} \mathrm{d} \Omega\left(\frac{1}{2} \mathrm{U}_{\mathrm{j}}^{\mathrm{w}} \mathrm{w}_{\mathrm{i}}^{\mathrm{n}}\right)\right.$ os quais substituidos em (6) conduzem aos valores das variáveis a nível de elemento, $\cup_{E}^{n+1 / 2}$, e ainda considerando que $P_{E}$ é uma função diferente de zero apenas no elemento, a Equação (6) pode ser escrita da seguinte forma:

$$
\begin{aligned}
& \Omega_{E}^{n+1 / 2} U_{E}^{n+1 / 2}=\left[\sum_{i}\left(\int_{\Omega} N_{j} \mathrm{~d} \Omega\right) U_{j}^{n}\right] \\
& -\frac{1}{2} \Delta t\left[\sum\left(\int_{\Omega} \frac{\partial N_{j}}{\partial x_{i}} d \Omega\right)\left(F_{i j}^{n}-R_{d i j}^{n}-\frac{1}{2} U_{j}^{n} w_{i}^{n}\right)\right]
\end{aligned}
$$

$\left.+\frac{1}{2} \Delta t \sum\left(\int_{n E} N_{j} d \Omega\right) R_{S_{i}}^{n}\right]$

onde $\Omega_{E}^{n+1 / 2}$ é a área do elemento no tempo $t_{n+1 / 2}$.

Segundo passo - No segundo passo de tempo o algoritmo calcula o vetor $U^{n+1}$ utilizando, novamente, uma expansão do vetor $U$ em série de Taylor, agora da forma:

$$
\mathrm{U}^{\mathrm{n}+1}=\mathrm{U}^{\mathrm{n}}+\left.\Delta \mathrm{t} \frac{\hat{\partial} \mathrm{U}^{\mathrm{n}+1 / 2}}{\partial \mathrm{t}}\right|^{-1}
$$

Considerando a Equação (3) podemos escrever a Equação (8) da seguinte forma:

$U^{n+1}=U^{n}-\Delta\left[\frac{\partial F_{i}^{n+1 / 2}}{\partial x_{i}}-\frac{\partial R_{d i}^{n+1 / 2}}{\partial x_{i}}-R_{s}^{n+1 / 2}-w_{i}^{n+1 / 2} \frac{\partial U^{n+1 / 2}}{\partial x_{i}}\right](9)$

onde as aproximações de elementos finitos utilizadas para as variáveis são:

$$
\begin{array}{ll}
U^{n+1} \equiv \sum_{j} U_{j}^{n+1} N_{j} ; & w_{i}^{n+1 / 2} \equiv \sum_{E} P_{E} w_{i_{E}}^{n+1 / 2} \\
F_{i}^{n+1 / 2} \equiv \sum_{E} P_{E} F_{i_{E}}^{n+1 / 2} ; & U^{n+1 / 2} \equiv \sum_{E} P_{E} U_{E}^{n+1 / 2} \\
R_{d i}^{n+1 / 2} \simeq \sum_{E} P_{E} R_{d_{E}}^{n+1 / 2} ; & R_{S}^{n+1 / 2} \simeq \sum_{E} P_{E} R_{S_{E}}^{n+1 / 2}
\end{array}
$$

Aplicando à (9) a forma residual ponderada de Galerkin e, considerando que:

$$
\begin{aligned}
& \Sigma\left(\int_{\Omega} N_{K} P_{E} \frac{\partial P_{E}}{\partial x_{i}} d \Omega\right) U_{E}^{n+1 / 2} w_{i E}^{n+1 / 2}= \\
& =\sum_{E}\left(\int_{\Omega} N_{K} \frac{\partial P_{E}}{\partial x_{i}} d \Omega\right) U_{E}^{n+1 / 2} w_{i_{E}}^{n+1 / 2}
\end{aligned}
$$


uma vez que $P_{E}$ é uma função constante associada com o elemento (Vaz dos Santos, 1993), obtémse os valores nodais requeridos de $U_{j}^{n+1}$ como:

$$
\begin{aligned}
& \sum_{j}\left(\int_{\Omega} \Omega^{2+1} \mathrm{~N}_{K} \mathrm{~N}_{j} \mathrm{~d} \Omega \mathrm{U}_{j}^{+1}=\sum_{j}\left(\int_{\Omega} \mathrm{N}_{\mathrm{K}} \mathrm{N}_{j} \mathrm{~d} \Omega \mathrm{U}_{j}^{n}\right.\right.
\end{aligned}
$$

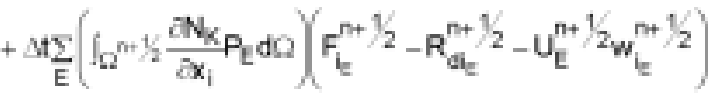

$$
\begin{aligned}
& +\Delta t \sum_{E}\left(h_{S}+1 / 2 N_{k} P_{E} d Q\right) R_{S}^{n+1 / 2} \\
& -\Delta \sum_{E}\left(G_{\Gamma}+1 v_{i} N_{k} P_{E} d \Gamma\right)\left(F_{i}^{n+1 / 2}-R_{d_{k}}^{n+1 / 2}-U_{E}^{n+1 / 2} w_{i \varepsilon}^{n+1 / 2}\right)
\end{aligned}
$$

A equação acima pode ser escrita na forma matricial compacta como:

$$
M^{n+1} U^{n+1}=M^{n} U^{n}+R_{\Omega}+S_{\Gamma}
$$

onde, $\mathrm{M}^{\mathrm{n}+1}$ é a matriz massa ao tempo $\mathrm{t}_{\mathrm{n}+1}, \mathrm{M}^{\mathrm{n}}$ é a matriz massa ao tempo $t_{n}, R_{\Omega}$ e $S_{\Gamma}$ representam, respectivamente, a contribuição das integrais de volume e superfície, como segue:

$$
\begin{aligned}
& M^{n+1}=\sum_{j}\left(b_{C}+1 N_{k} N_{i} d \Omega\right) \\
& M^{n}=\sum_{\mathrm{i}}\left(\mathrm{C}_{2}+\mathrm{N}_{k} \mathrm{~N}_{\mathrm{j}} \mathrm{en}\right)
\end{aligned}
$$

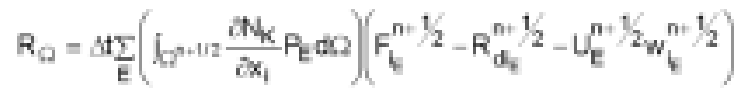

$$
\begin{aligned}
& +\Delta \sum_{E}\left(f_{n}+v_{2} N_{k} P_{E} d \Omega R_{\mathrm{s}}^{n-1 / 2}\right. \\
& S_{T}=-M \sum_{E}\left(S_{\Gamma} I_{1} N_{k} P_{E} d \Gamma\right)\left(F_{t}^{n+1 / 2}-R_{d e}^{n+1 / 2}-U_{E}^{n+1 / 2} w_{t}^{n+1 / 2}\right)
\end{aligned}
$$

A avaliação das expressões integrais para $\mathrm{R}_{\Omega}$ e $\mathrm{S}_{\Gamma}$ requer os valores das variáveis de campo $F_{i}^{n+1 / 2}, R_{d}^{n+1 / 2}$ e $R_{S}^{n+1 / 2}$ para o instante intermediário de tempo $t=t_{n+1 / 2}$. Em $R_{\Omega}$ as integrais são avaliadas no domínio do elemento $\mathrm{E}, \mathrm{em}_{\Gamma}$ as integrais são avaliadas sob a superfície em conjunto com a normal externa local dos respectivos elementos. Como conseqüência do primeiro passo do algoritmo, as variáveis dependentes para o tempo inter- mediário são obtidas apenas em valores médios nos elementos. Assim:

$$
\begin{aligned}
& F_{i}^{n+1 / 2}=F_{i}\left(U^{n+1 / 2}\right) \\
& R_{d_{i}+1 / 2}^{n+1 / 2}=R_{d_{i}}\left(U^{n+1 / 2}\right) \\
& R_{S}^{n+1 / 2}=R_{S_{i}}\left(U^{n+1 / 2}\right)
\end{aligned}
$$

usam o valor do vetor $U^{n+1 / 2}$, obtido no primeiro passo. Este procedimento é satisfatório na avaliação das integrais de volume. No entanto, para a avaliação das integrais de superfície em $\mathrm{S}_{\Gamma}$ uma correção deve ser feita no cálculo das variáveis de campo obtidas através de (15) (Vaz dos Santos, 1993).

O algoritmo proposto estima os valores de contorno das variáveis de campo aparecendo nas integrais de superfície em $\mathrm{S}_{\mathrm{r}}$ da forma:

$$
\begin{aligned}
& F_{i_{s}}^{n+1 / 2}=F_{i_{s}}^{n}+F_{i}^{n+1 / 2}-F_{i}^{n} \\
& R_{d_{s}}^{n+1 / 2}=R_{d_{s}}^{n}+R_{d_{i}}^{n+1 / 2}-R_{d_{i}}{ }^{n}
\end{aligned}
$$

onde o subíndice S refere-se aos valores de contorno das variáveis de campo.

A Equação (13) é resolvida explícita e iterativamente conforme proposto por-Lohner et al., (1985) como segue:

$$
M_{L}^{n+1} U_{K+1}^{n+1}=M^{n} U^{n}+R_{\Omega}+S_{\Gamma}-\left[M^{n+1}-M_{L}^{n+1}\right] \mu_{K}^{n+1}
$$

onde, $\mathrm{k}$ indica a k-ésima iteração, $\mathrm{M}_{\mathrm{L}}^{\mathrm{n}+1}$ é a matriz de massa discreta em $t_{n+1}$.

A fórmula de recorrência expressa na Equação (17) utiliza a matriz massa discreta $\mathrm{M}_{\mathrm{L}}$ do sistema como uma matriz de iteração e, requer como consequência, exclusivamente operações vetoriais (Vaz dos Santos,1993). Normalmente, um pequeno número de iterações é suficiente para atingir a convergência no intervalo.

Sendo o método de Taylor-Galerkin de dois passos um método explícito fica sujeito à condição de estabilidade de Courant (Lõhner et al., 1985). Tal condição aplicada sobre um elemento E estabelece que o passo de tempo local $\Delta \mathrm{t}_{\mathrm{E}}$ deve satisfazer a desigualdade:

$$
\Delta t_{E} \leq \frac{\beta h_{E}}{\max _{E}(\mid u+c)}
$$


onde, u é a velocidade do fluido, c a celeridade da onda, $\mathrm{h}_{\mathrm{E}}$ um comprimento característico do elemento e $\beta=1 / \sqrt{3}$ é um fator de segurança aplicado ao número de Courant (Löhner et al., 1985).

\section{Condições de contorno, viscosidade artificial e atualização da malha}

A imposição das condições iniciais requer o conhecimento da superfície livre para o tempo $\mathrm{t}=0$, o que nem sempre é possível, adotando-se a condição de elevação zero como regra geral. Com relação às condições de contorno, como as tensões de fricção no fundo e de cisalhamento na superfície livre prevalecem sobre as tensões laterais, é imposta a condição de fluxo paralelo aos contornos sólidos, isto é, as velocidades normais são nulas e as velocidades tangenciais são deixadas livres. Para os contornos de entrada são prescritas as velocidades e elevação da superfície livre, enquanto que nos contornos de saída as variáveis são deixadas livres.

A fim de estabilizar numericamente a solução na presença de choques, utiliza-se um modelo de viscosidade artificial, o qual dissipa sobre os elementos vizinhos os altos gradientes. Este amortecimento é adicionado explicitamente à solução de forma a reduzir o tempo de CPU.

A atualização das coordenadas da malha de elementos finitos é feita com base no procedimento proposto por Donea et al. (1982), de forma a garantir a eficiência do procedimento frente à arbitrariedade da velocidade da malha.

\section{APLICAÇÕES}

\section{Embancamento de uma onda}

O problema analisado simula o avanço de uma onda solitária em direção a uma praia.

Quando ondas se propagam sobre águas progressivamente mais rasas têm, via de regra, sua altura aumentada e o comprimento diminuído. A senoidal que representa o movimento oscilatório simples fica cada vez mais deformada e assimétrica, as cristas tornam-se anguiares e íngremes seguidas de cavas praticamente planas. Chegará um ponto em que esta assimetria será marcante a ponto de comprometer a estabilidade do próprio movimento, ocorrendo a quebra das ondas.

Devido à característica assimétrica do movimento de uma onda chegando a uma praia, a aplicabilidade das teorias de onda de Airy e de
Stokes é restrita, tornando-se necessário a utilização da teoria de onda solitária (Dean, 1991).

A onda solitária é uma onda composta de uma única crista propagando-se pelo oceano; não há período ou comprimento de onda. A velocidade de propagação da onda solitária está relacionada apenas com a profundidade da água. Assim, quando as ondas solitárias se propagam em águas rasas apresentam forte similaridade com as ondas reais. Por esse motivo, as ondas solitárias são utilizadas para representar os fenômenos próximos à costa.

Na Figura 1 é mostrado a variação da profundidade do domínio em estudo, bem como a equação da onda e as condições iniciais para $t=0$, dadas por:

$$
\begin{gathered}
\eta=a_{0} \operatorname{sech}^{2} 0.5\left(3 a_{0}\right)^{0,5}\left(x-\alpha^{-1}\right) \\
u=-\left(1=0.5 a_{0}\right) h /(\alpha x+\eta) \\
a_{0}=0.1 g=1.0 \alpha=1 / 30 \\
L=40
\end{gathered}
$$

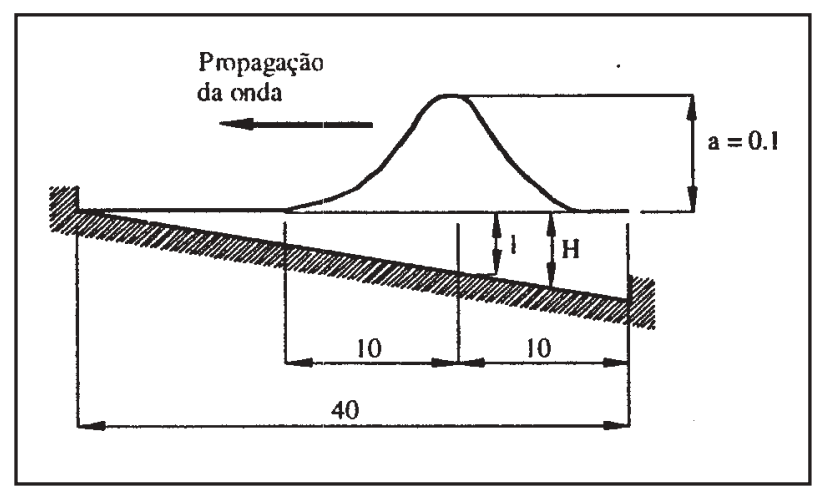

Figura 1. Variação linear da batimetria e a elevação da onda em $t=0$.

Neste trabalho, mostra-se que o algoritmo implementado representa o que foi citado anteriormente. E ainda, segundo o princípio de conservação da energia, a diminuição da profundidade e consequentemente da velocidade de propagação da onda, provoca um aumento na altura da onda, o que caracteriza o processo de embancamento ou "shoaling", como mostra a Figura 2.

A malha de elementos finitos utilizada tem 205 nós e 320 elementos triangulares. O passo de tempo empregado é de $\Delta t=0,125 \mathrm{~s}$.

A Figura 2 mostra os valores de elevação, para três instantes de tempo, obtidos pelo algoritmo proposto, e a Figura 3 os valores segundo 


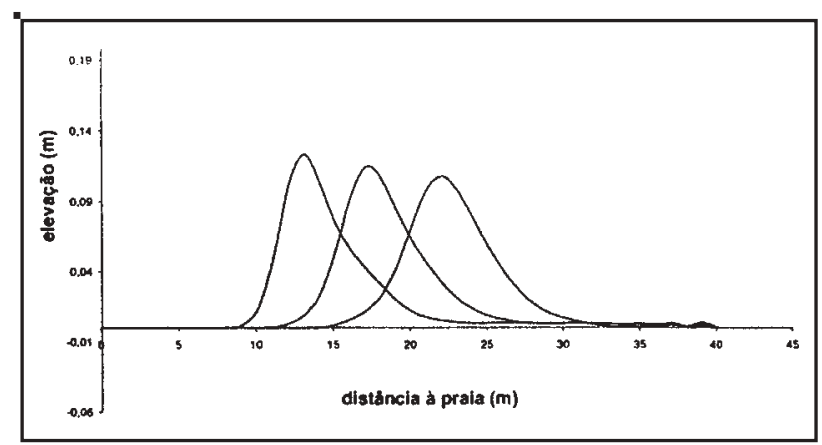

Figura 2. Embancamento de uma onda solitária para 60, 100 e $140 \Delta t$ e $C_{C}=0$.

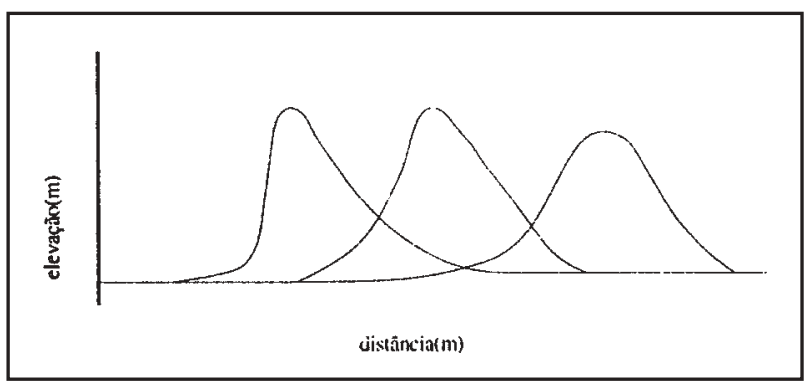

Figura 3. Resultados obtidos por Lönher et al. (1984).

Löhner et al. (1984). A Figura 4 mostra o "shoaling" de uma onda para 8 intervalos de tempo de $20 \Delta$ t (segundos), sem amortecimento artificial.

O algoritmo proposto reproduz de maneira satisfatória o embancamento de uma onda. Cabe salientar que o domínio em estudo é fechado e o avanço da onda foi produzido até o ponto anterior ao de quebra (arrebentação).

\section{Ruptura de uma barragem}

O problema unidimensional simula o rompimento de uma barragem que separa dois níveis distintos de água em repouso, num canal de seção constante (Peraire et al., 1986).

A barragem é bruscamente rompida e ondas íngremes progridem dentro dos dois domínios. A simulação foi efetuada com uma malha de 205 nós e 320 elementos triangulares e um passo de tempo $\Delta \mathrm{t}=0,25 \mathrm{~s}$. As condições iniciais dadas são $U_{1}=0 ; \eta=1,0 \leq x \leq 20 ; \eta=0,20 \leq x \leq 40$, onde $U_{1}$ é a componente da velocidade de escoamento na direção horizontal, $\eta$ é a elevação da superfície, $H$ é a profundidade em relação ao nível de referência e g a aceleração da gravidade.

A Figura 5 mostra os perfis obtidos para a elevação da superfície e velocidade em três tem-

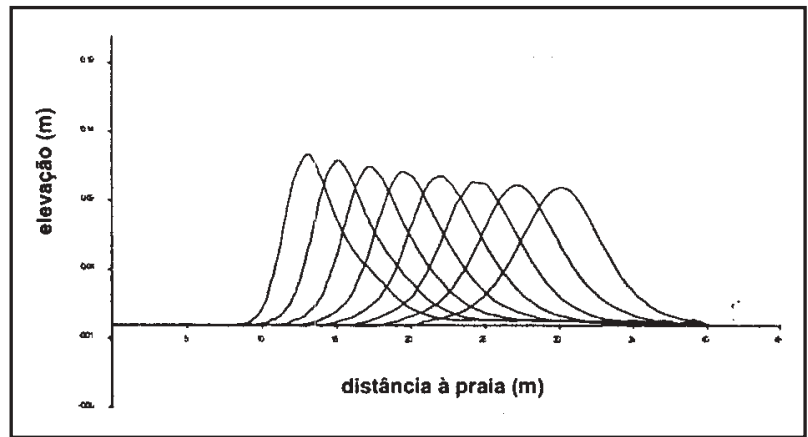

Figura 4. Embancamento de uma onda para 8 intervalos de $20 \Delta t$ e $C_{C}=0$.
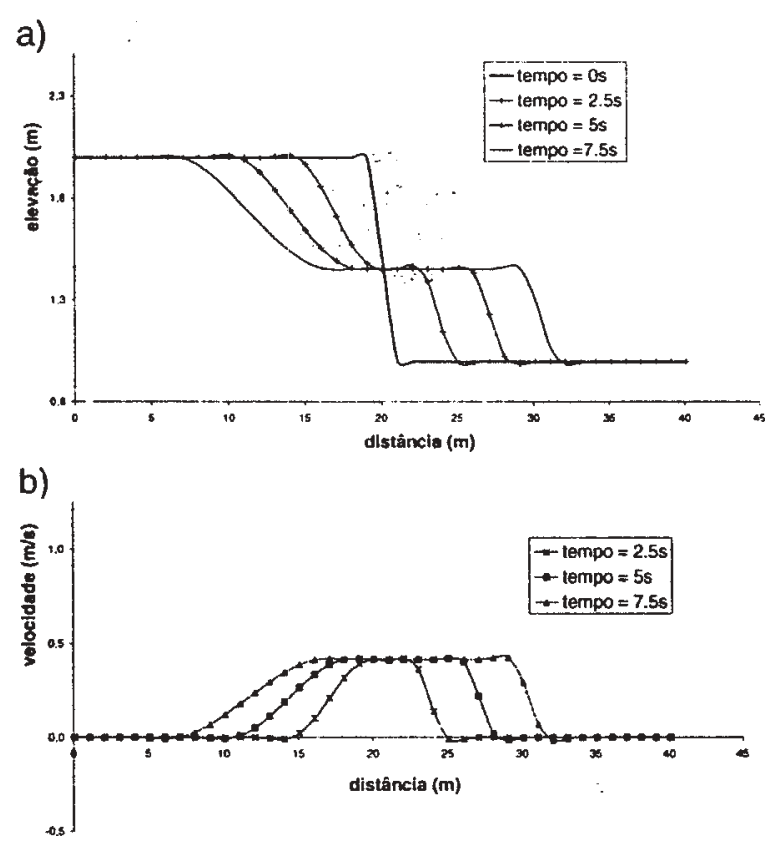

Figura 5. Ruptura de uma barragem: a) elevação da superfície da água e b) velocidades.

pos $(10 \Delta t, 20 \Delta t$ e $30 \Delta t)$, com amortecimento artificial $\mathrm{C}_{\mathrm{c}}=1$.

A Figura 6 mostra os resultados obtidos sem amortecimento artificial. Nota-se uma certa frente íngreme na parte rasa e a diminuição do gradiente para a região mais profunda.

Os resultados obtidos são de boa qualidade, comparados com os, resultados apresentados por Löhner et al. (1984), mostrados na Figura 7.

Nota-se, ao comparar os resultados apresentados na Figura 6 com os resultados mostrados na Figura 5, que a introdução do amortecimento atenua a frente íngreme na parte rasa e as oscilações numéricas. 


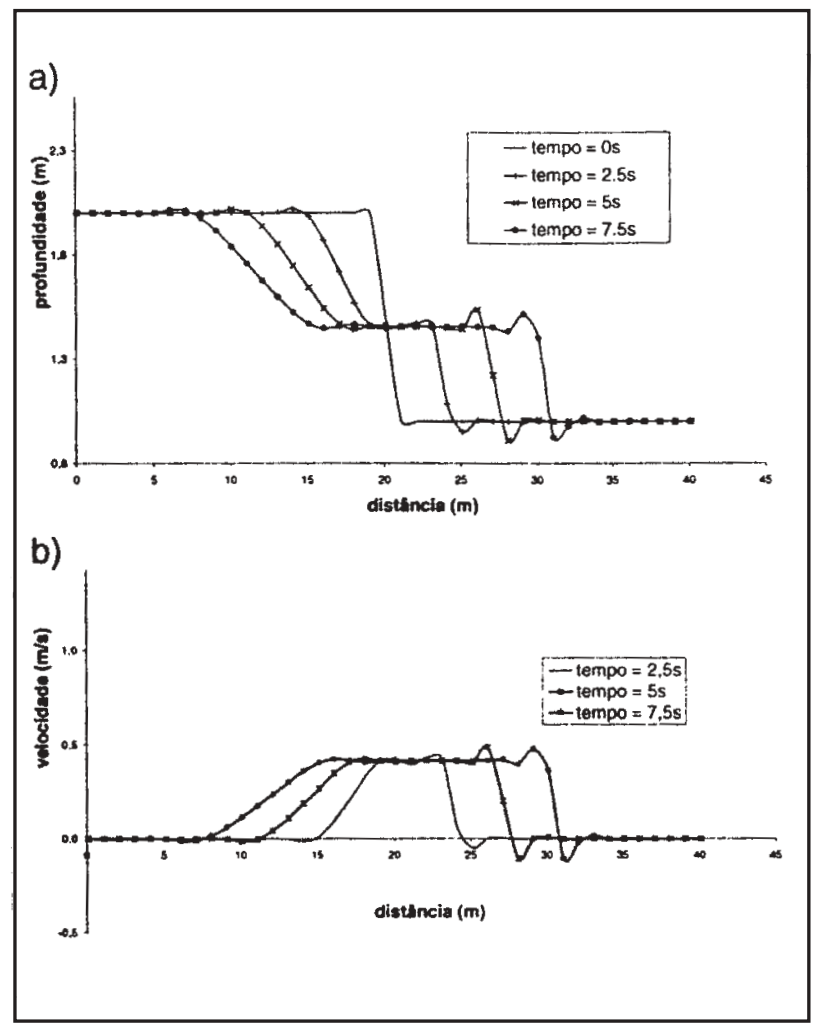

Figura 6. Ruptura de uma barragem: a) elevação da superfície da água e b) velocidades, $\mathrm{C}_{\mathrm{C}}=0$.

a)

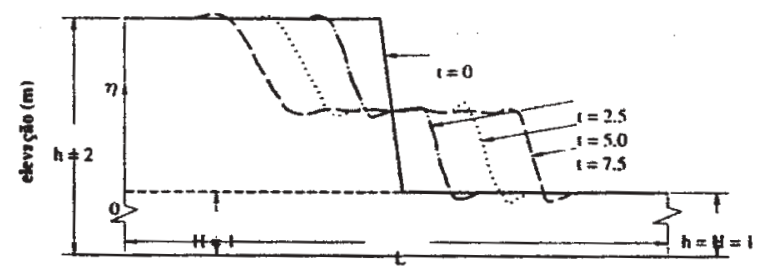

b)

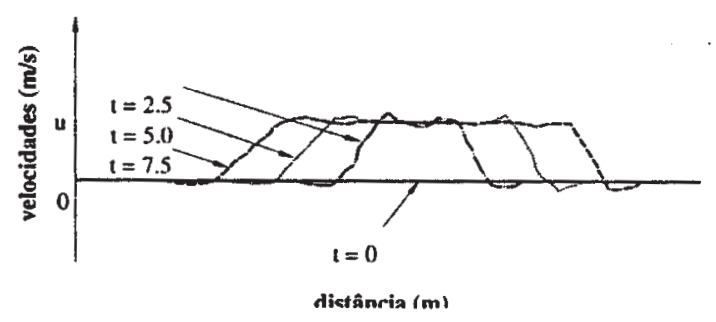

Figura 7. Perfis da elevação da superfície a) e da velocidade b) para três tempos distintos, segundo Löhner, Morgan e Zienkiewicz (1984).

\section{Descarga de um rio}

O problema apresentado simula a formação de barras d'água, em um rio, provocadas pelo movimento da maré (Peraire et al., 1986). É analisada a formação de uma pororoca idealizada, progredindo dentro de um canal, que carrega água com velocidade uniforme, causada pelo aumento gradual do nível d'água a jusante. A configuração inicial é dada por: $U_{1}=1.0 ; H=1.0 ; \eta=0$; $g=0.667$ (valores adimensionais). A elevação da onda, à esquerda do domínio, é incrementada conforme função indicada na Figura 8.

A malha utilizada possui 123 nós e 160 elementos triangulares, com um passo de tempo $\Delta \mathrm{t}=0,22$.

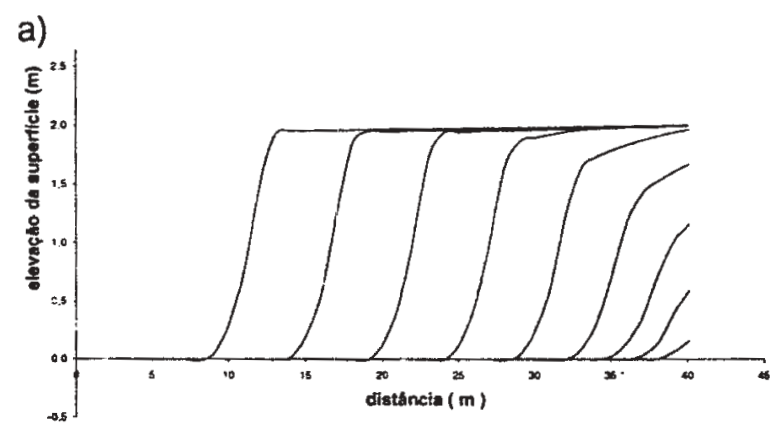

b)

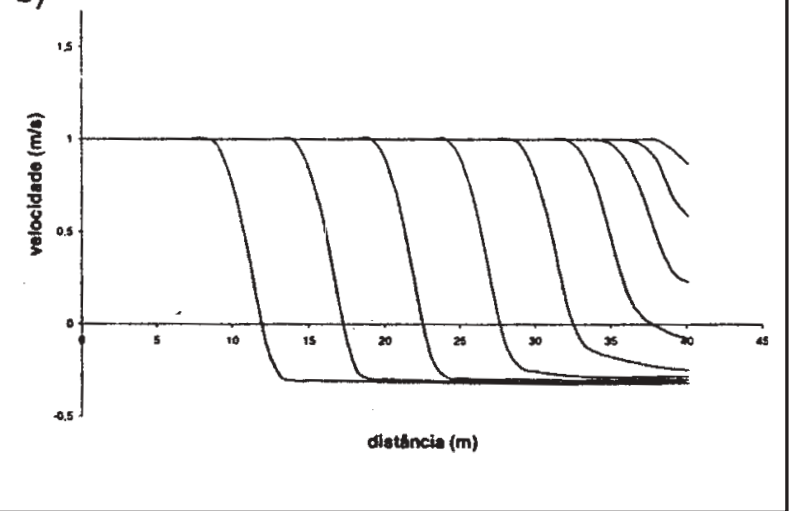

Figura 8. Barra d’água em um curso d'água, devido ao aumento gradual do nível a jusante: a) elevação da superfície e b) velocidade em intervalos de $25 \Delta t . \eta=1-\cos \left(\pi t / T_{0}\right)$ para $0 \leq \mathrm{t} \leq 30, \eta=2$ para $\mathrm{t} \geq 30$.

Apesar do fluxo ser subcrítico, nota-se um aumento na altura da barra d'água desenvolvendo claramente um choque. Para a análise deste problema foi necessário adicionar o modelo de viscosidade artificial. O aumento do coeficiente de 
amortecimento artificial $\mathrm{C}_{\mathrm{c}}$ atenua de maneira acentuada a frente de choque, Figura 8 . Os resultados obtidos estão em concordância com os resultados apresentados por Zienkiewicz et al. (1985), como mostram as Figuras 9 e 10.

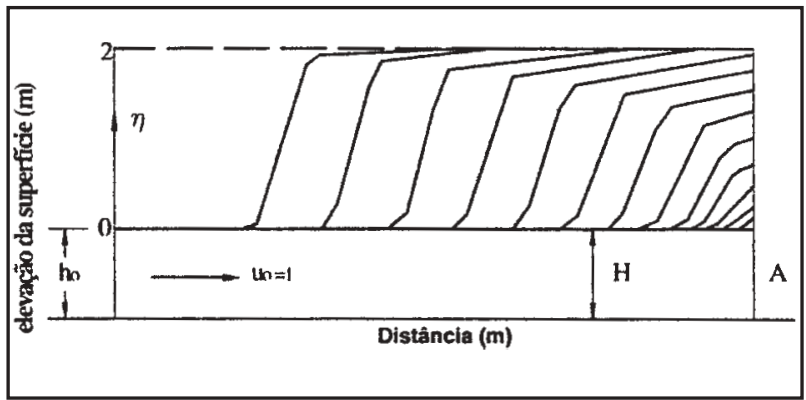

Figura 9. Elevação da superfície segundo Zienkiewicz et al. (1985).

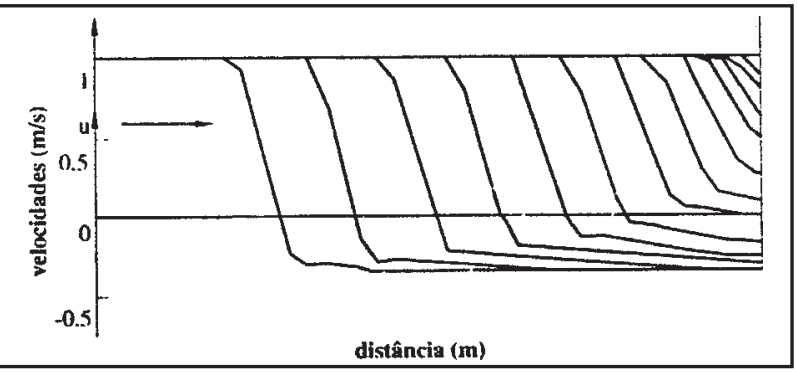

Figura 10. Velocidades apresentadas por Zienkiewicz et al. (1985).

\section{Canal retangular com restrição}

É analisado o escoamento bidimensional supercrítico em um canal (Peraire et al., 1986).

O problema consiste de um canal de profundidade uniforme de $1 \mathrm{~m}$, aberto nas extremidades, que tem' sua seção transversal reduzida abruptamente por um degrau perpendicular ao escoamento não perturbado.

O escoamento inicial foi prescrito como supercrítico para todos os elementos do domínio com número de Froude de 2.92.

A solução em regime permanente é obtida após, aproximadamente, 500 passos de tempo. 0 problema é resolvido utilizando uma malha de elementos finitos triangulares, composta de 288 elementos e 171 nós, com passo de tempo $\Delta t=0,075 \mathrm{~s}$.

As condições de contorno foram impostas diferenciadamente para a quina, onde o nó é deixado livre, e para o canto do degrau, onde as duas componentes da velocidade são consideradas nulas, em relação aos demais pontos onde a velocidade normal prescrita é nula.
Na Figura 11 são apresentados, respectivamente, as soluções relativas ao campo de velocidades, as isolinhas de profundidade e a profundidade d'água ao longo do eixo central do canal.

a)

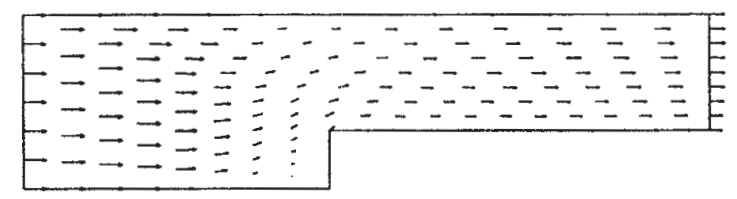

b)

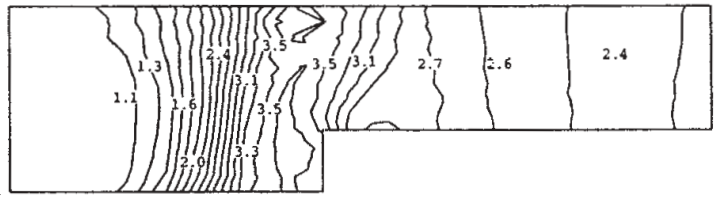

c)

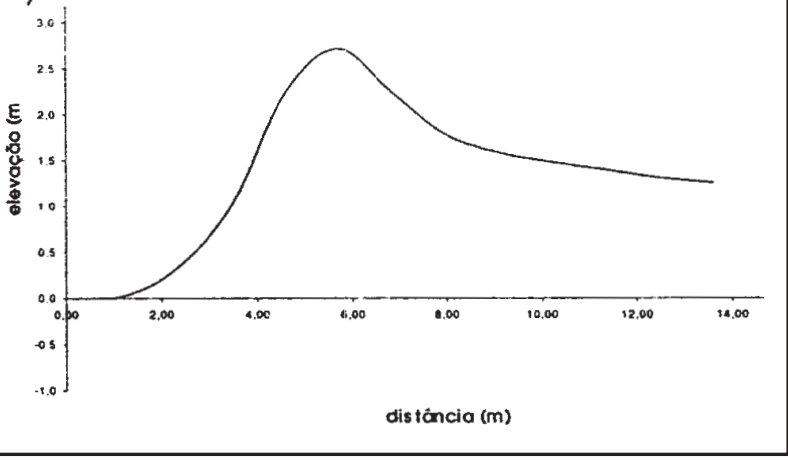

Figura 11. a) Campo de velocidades para o canal com restrição, b) isolinhas de profundidade e c) elevação da superfície da água.

Analisando os resultados obtidos na Figura 11 observa-se que, na seção transversal reduzida abruptamente pelo degrau, o escoamento produz uma onda de choque ou salto hidráulico, que,pode ser visto no gráfico das isolinhas de profundidade e no gráfico da profundidade d'água no centro do canal. Os resultados obtidos nesse trabalho. são coerentes com os apresentados por Peraire et al. (1986), mostrados na Figura 12.

\section{Propagação de uma onda longa num canal retangular com dissipação}

Nesta aplicação, uma forma senoidal da elevação da superfície, dada por $\eta=\eta_{0} \operatorname{sen}(2 \pi \mathrm{t} / \mathrm{T})$, 


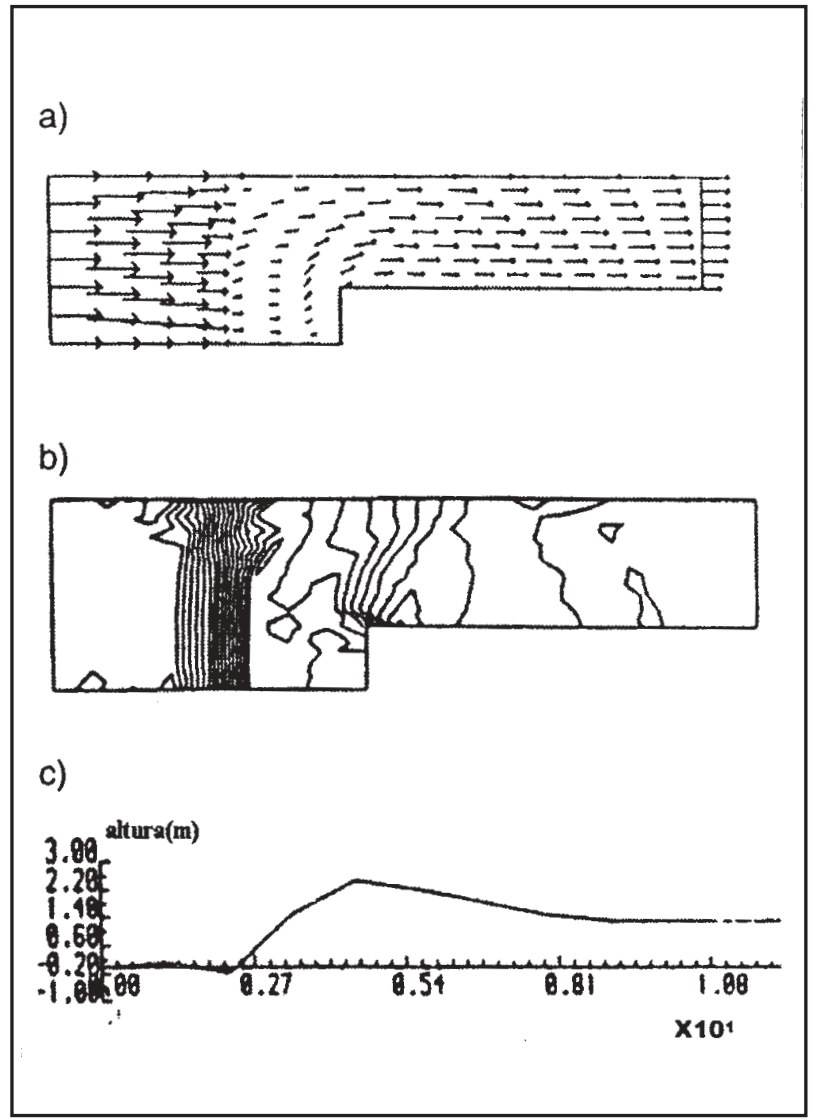

Figura 12. Resultados segundo Peraire et al. a) Camp de velocidades, b) isolinhas de profundidade e c) elevação.

é aplicada ao contorno externo aberto de um canal longo retangular com atrito dissipativo no fundo, fechado na outra extremidade. e 115 nós.

É utilizada uma malha com 176 elementos

São dados: a amplitude da onda $\eta=0.1 \mathrm{~m}$; período $\mathrm{T}=12 \mathrm{~h}$; coeficiente de Chezy $\mathrm{C}=50 \mathrm{~m} / \mathrm{s}^{1 / 2}$; aceleração da gravidade $\mathrm{g}=9,81 \mathrm{~m} / \mathrm{s}^{2} ;$ pressão atmosférica $\mathrm{p}_{\mathrm{a}}=103320 \mathrm{~Pa}$; massa específica $\rho=1000 \mathrm{~kg} / \mathrm{m}^{3}$; profundidade $\mathrm{H}=10 \mathrm{~m}$. O passo de tempo empregado é de $\Delta \mathrm{t}=\mathrm{T} / 40 \mathrm{~s}$.

A Figura 13 mostra os resultados em regime permanente obtidos pelo modelo proposto, após 5 a 6 ciclos, bem como a solução analítica (Lee et al., 1987).

Analisando os resultados mostrados na $\mathrm{Fi}$ gura 13 conclui-se que o modelo desenvolvido neste trabalho é eficaz, pois a solução numérica obtida aproxima-se da solução analítica apresentada por Lee et al. (1987). Cabe salientar que os valores na referência foram analisados considerando um modelo de fricção no fundo linear, diferente do modelo de fricção aqui utilizado.

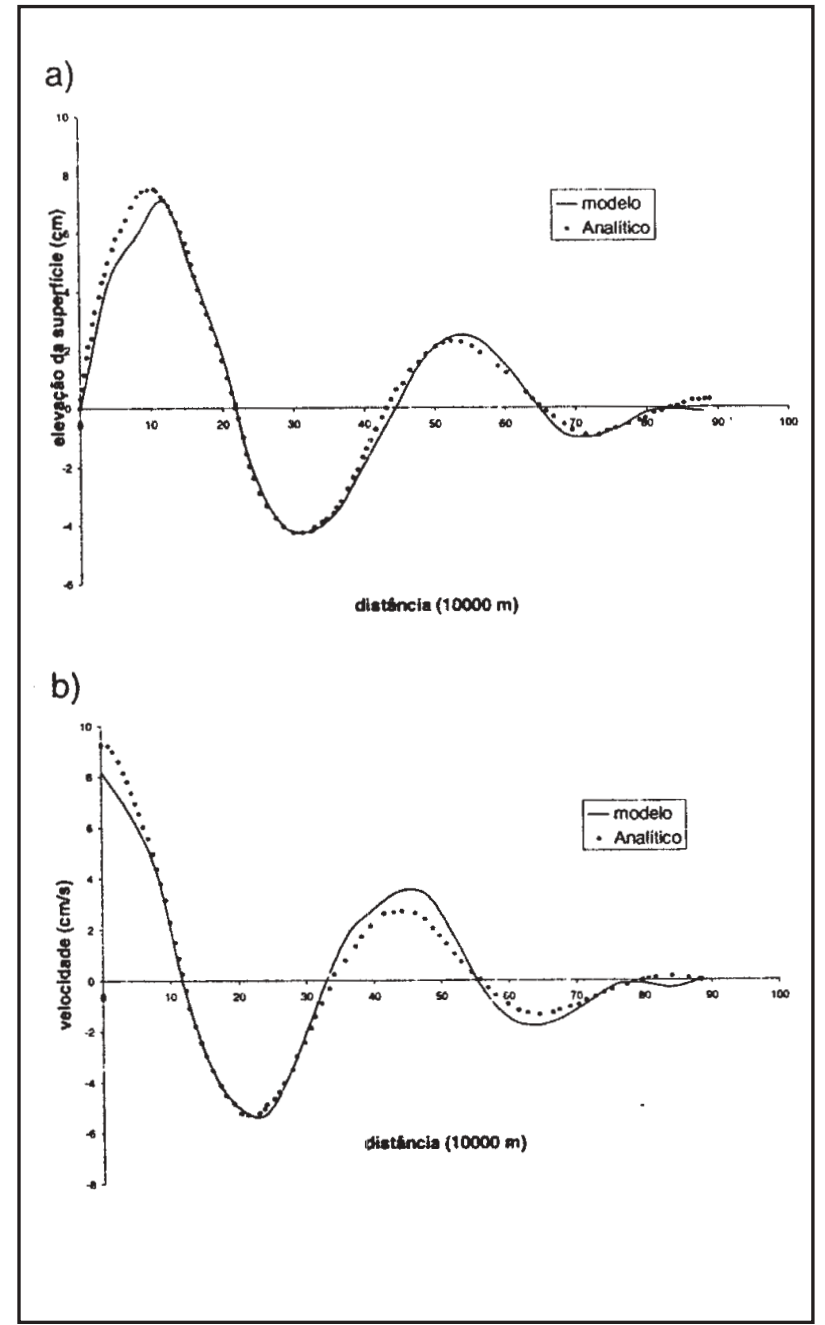

Figura 13. Canal com fricção no fundo: a) elevação em $T / 2$ e b) velocidade em $3 T / 4$.

\section{Canal com contorno móvel}

Este exemplo introduz o movimento da malha de elementos finitos que torna o contorno do domínio móvel, simulando as variações na elevação da água induzidas por movimentos de maré em uma praia inclinada (Awruch, 1983).

Nos exemplos anteriores os contornos foram considerados fixos, impondo ali a condição de que o fluxo seja nulo na direção normal ao contorno. Dessa maneira está sendo imposta uma condição não realística no fim e/ou no início do domínio. Então, para conhecer a real posição do contorno e não violar a equação da continuidade, é suficiente que o contorno se mova com velocidade igual a da componente normal da velocidade do fluido, isto é, o produto do vetor unitário normal ao contorno pela diferença entre as velocidades do fluido e do contorno deve ser nulo. 
A implementação prática da descrição arbitrária lagrangeana-euleriana (ALE) requer um procedimento de atualização da malha de elementos finitos automático e contínuo, de maneira a assegurar que o movimento arbitrário prescrito para o domínio de referência seja sempre adequado ao problema particular em estudo. O modelo utiliza um procedimento de rezoneamento automático e contínuo da malha, onde a idéia básica é dar ao nó em estudo uma velocidade da malha atualizada, igual a média das velocidades da malha em relação aos nós vizinhos para o passo de tempo anterior. A arbitrariedade na escolha da velocidade da malha deve ser tratada com cuidado, a fim de garantir a eficiência do método.

Para a simulação do movimento da maré em uma praia, é considerado um domínio com um contorno aberto, outro móvel e os outros dois fechados.

As profundidades médias variam linearmente na direção longitudinal com o máximo de 9 metros na entrada, onde é imposta a elevação da onda provocada pela maré.

A condição de velocidade normal zero foi aplicada ao longo das paredes e uma elevação senoidal de período $12 \mathrm{~h}$ e amplitude $0.2 \mathrm{~m}$ foi prescrita no contorno de entrada. Para o contorno de saída, as variáveis foram deixadas livres e a velocidade da malha foi prescrita de forma a acompanhar as variações na elevação, devido ao movimento de maré.

A Figura 14a mostra a malha de elementos finitos, composta de 72 elementos triangulares e 50 nós e $\Delta x=15 \mathrm{~km}, \Delta y=15 \mathrm{~km}$. O coeficiente de Chezy adotado é de $50 \mathrm{~m}^{1 / 2} / \mathrm{s}$ para os nós com até 3 metros de profundidade, $40 \mathrm{~m}^{1 / 2} / \mathrm{s}$, para os nós com até 2 metros de profundidade e $30 \mathrm{~m}^{1 / 2} / \mathrm{s}$ para os nós com menos de 2 metros, conforme Awruch (1983).

Os valores do coeficiente de Chezy foram escolhidos de forma'a levar em consideração a influência da profundidade na resistência do fundo ao escoamento.

As Figuras 14b e 14c mostram, respectivamente, o movimento do contorno móvel e a elevação da linha com $1 \mathrm{~m}$ de profundidade para o terceiro e quarto ciclo. O contorno se desloca com o mesmo período da maré. A elevação dos nós vizinhos ao contorno móvel, $\mathrm{H}=1 \mathrm{~m}$, oscila também com o mesmo período.

Os resultados obtidos mostram a capacidade do modelo em resolver problemas levando em consideração as variações nas linhas de costa em função de oscilações da maré.

\section{CONCLUSÕES}

O modelo matemático formulado e implementado neste trabalho, simula de maneira satisfatória escoamentos bidimensionais de fluidos incompressíveis, usando uma formulação geral das a)

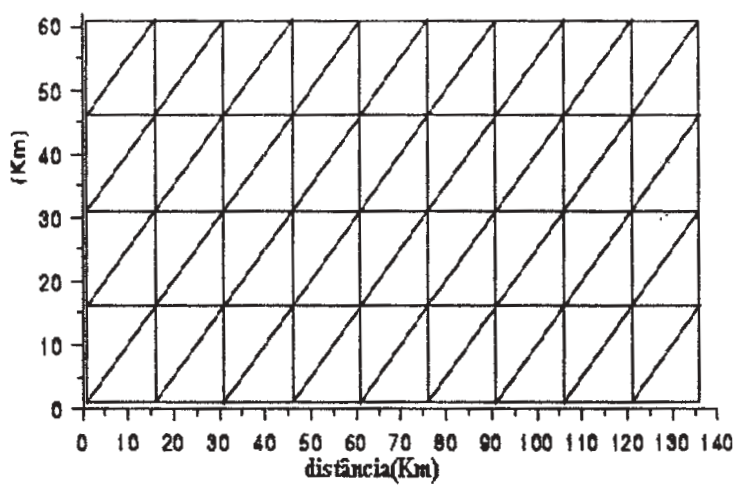

b)

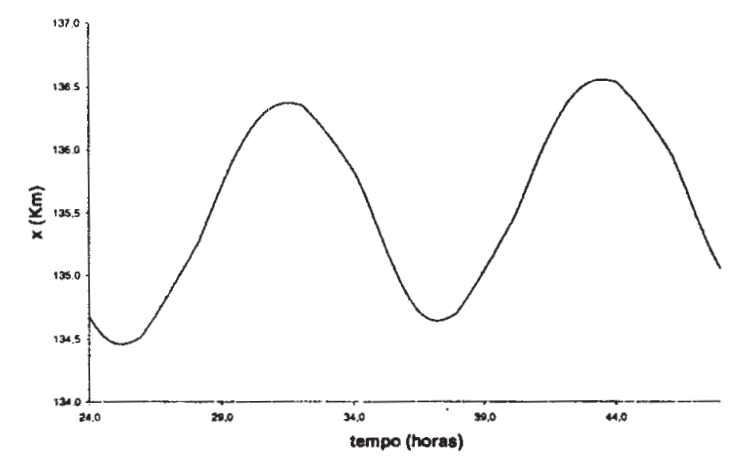

c)

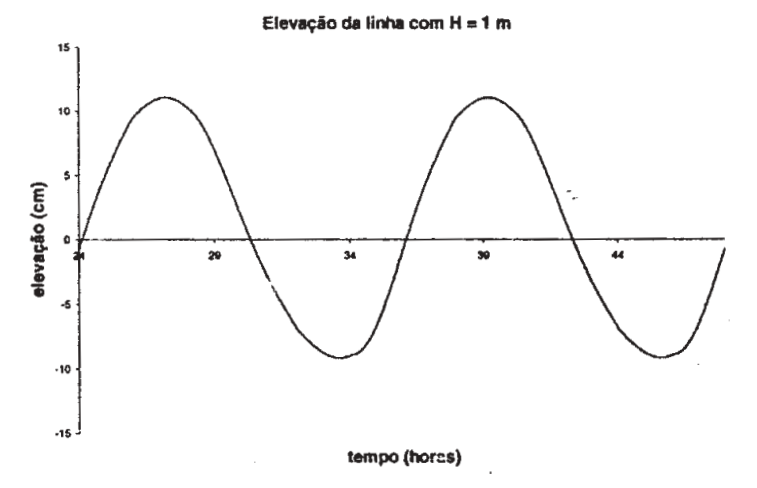

Figura 14. Canal com contorno móvel: a) malha de elementos finitos, b) movimento do contorno móvel e c) elevação da linha com $\mathrm{H}=1$ m.

equações de águas rasas na sua forma conservativa, e explorando a similaridade das mesmas com as equações para escoamento de fluidos compressíveis

A forma explícita do método de TaylorGalerkin de dois passos facilita a sua implementação vetorial, tornando direta a adição de equações de transporte hidrodinâmico, permitindo a modelagem de 
efeitos adicionais tais como, dispersão de poluentes ou distribuição de temperatura.

A viscosidade artificial estabiliza numericamente a solução de problemas envolvendo escoamentos supercríticos, onde tem-se oscilações nas proximidades dos saltos hidráulicos.

A descrição arbitrária lagrangeanaeuleriana (ALE) permite a malha de elementos finitos acompanhar os contornos móveis, como por exemplo, nas oscilações induzidas por maré, ou ainda acompanhar o movimento de estruturas imersas no fluido. A arbitrariedade na escolha da velocidade da malha deve ser tratada com cuidado, a fim de garantir a eficiência do método.

O modelo implementado utiliza um método explícito que está sujeito à condição de estabilidade de Courant, a qual restringe o passo de tempo.

Pode-se concluir que o modelo formulado e implementado é eficiente, quando aplicado a problemas de escoamentos em corpos d'água rasos.

\section{REFERÊNCIAS}

AWRUCH, A. M. (1983). Introdução de Contornos Móveis na Solução das Equações de Águas Rasas Através de Elementos Finitos, COBEM 83, p295-304.

BURNETT, D. (1987). Finite Element Analysis, Addison-Wesley Publishing Company

DONEA, J.; GIULIANI, S.; HALLEUX, J. P. (1982). An Arbitrary Lagrangian-Eulerian Finite Element Method for Transient Dynamic FluidStruture Interactions, Computer Methods in Applied Mechanicas and Engineering, v.33, p680-723.

DEAN, R. G.; DALRYMPLE, R. A. (1991). Water Wave Mechanics for Engineers and Scientists, World Scientific Publishing.

KAWAHARA, M.; HIRANO. TAKEUCHI, N. (1978). Two Step Explict Finite Element Method for Tsunami Wave Propagation Analysis, International Journal for Numerical Methods in Engineering, v.12, p331-351.

LEE, J. H. W.; PERAIRE, J. \& ZIENKIEWICZ, O. C. (1987). The Characteristic - Galerkin Method for Advection-Dominated Problems - An Assessment, Computer Methods in Applied Mechanics and Engineering, v.61, p359-369.

LOHNER, R.; MORGAN, K.; ZIENKIEWICZ, O. C. (1985). An Adaptative Finite Element Procedure for Compressible High Speed Flows, Computer Methods in Applied Mechanics and Engineering, v.51, p441-465.

LOHNER, R.; MORGAN, K.; ZIENKIEWICZ, O. C. (1984). The Solution of Non-Linear Hiperbolic
Equation Systems by the Finite Element Method, International Journal for Numerical Methods in Engineering, v.4, p1043-1063.

PERAIRE, J.; ZIENKIEWICZ, O. C.; MORGAN, K. (1986). Shallow water problems: A general explicit formulation, International Journal for Numerical Methods Engineering, v.22, p547574.

VAZ DOS SANTOS, M. A. (1993). Simulação Numérica de Escoamentos Compressíveis e sua Interação com Estruturas Deformáveis, Tese de Doutorado, UFRGS.

ZIENKIEWICZ, O. C.; LOHNER, R.; MORGAN, K. \& PERAIRE, J. (1985). High-Speed Compressible Flow and Other Advection Dominated Problems of Fluid Dynanics, Finite Element in Fluids, v.6, cap. 2, John Wiley \& Sons Ltda.

\section{An Explicit Formula to Solve Shallow Water Flows}

\section{ABSTRACT}

A numerical algorithm to simulate twodimensional incompressible flows, using a conservative general formula for shallow water equations is presented in this study. The model solves several coastal and environmental engineering problems including the study of shallow water navigation, the determination of pollutant dispersion, the analysis of dynamic behaviour of harbour structures and the determination of pollutant dispersion, the analysis of dynamic behaviour of harbour structures and the prediction of tides, currentes, and waves effects. The finite element technique is used for the spatial discretization of the fluid domain.

The algorithm is based on the TaylorGalerkin explicit procedure for time integration, which explores conservative properties of governing equations, and includes the accurate treatment of convective terms. An Arbitrary Euler-Lagrange (ALE) description is also employed optionally.

Some examples are analysed in order to show model behaviour and to validate its reliability and performance. 